\title{
Model-Based Transfer Entropy Analysis of Brain-Body Interactions with Penalized regression techniques*
}

\author{
Yuri Antonacci ${ }^{1,2}$, Laura Astolfi ${ }^{1,2}$, Alessandro Busacca ${ }^{3}$, Riccardo Pernice ${ }^{3}$, Giandomenico Nollo ${ }^{4}$ \\ and Luca Faes ${ }^{3}$
}

\begin{abstract}
The human body can be seen as a functional network depicting the dynamical interactions between different organ systems. This exchange of information is often evaluated with information-theoretic approaches which comprise the use of vector autoregressive (VAR) and state space (SS) models, normally identified with the Ordinary Least Squares (OLS). However, the number of time series to be included in the model is strictly related to the length of data recorded thus limiting the use of the classical approach. In this work, a new method based on penalized regressions, the so-called LASSO, was compared with OLS on physiological time-series extracted from 18 subjects during different stress conditions. Results show similarities between the brain-body interactions estimated by both methodologies, highlighting a greater intepretability of patterns estimated with LASSO especially in the subnetwork of brain-brain interactions.
\end{abstract}

\section{INTRODUCTION}

Recent studies have highlighted that the human body is an integrated network composed by different organ systems continuously interacting with each other. Studying these interactions could be very important because it has been demonstrated how the failure of a single system can cause the collapse of the whole network [1]. Network Physiology aims to study the mechanisms through which diverse physiological systems and organs dynamically interact to generate different physiological states. Novel methodologies have been introduced and tested in order to study the couplings between dynamic systems, e.g. cardiovascular and cardiorespiratory systems, or between brain and heart [2]. A possible approach for the evaluation of such information exchange relies on the identification of a vector autoregressive model (VAR) in a multivariate fashion, by means of ordinary least square estimator (OLS), and a state space (SS) model [3]. However, from a methodological point of view, the amount of data needed for such analysis increases exponentially with the number of processes analyzed jointly, thus imposing a limitation to the size of the network to be investigated. A solution is represented by

*Research supported by Progetti di Ateneo 2017 and 2018 (RM11715C82606455 - RM11916B88C3E2DE) and by Progetti di Avvio all Ricerca 2019

${ }^{1,2}$ Y. A.and L. A. are with the Department of Computer, Control and Management Engineering, University of Rome Sapienza and with IRCCS Fondazione Santa Lucia, Rome, Italy. yuri.antonacci@uniromal.it

${ }^{3}$ A. B., R. P. and L.F. are with the Department of Engineering, University of Palermo, Italy

${ }^{4}$ G. N. is with the Department of Industrial Engineering, University of Trento, Italy

978-1-7281-5751-1/20/\$31.00 @2020 IEEE the least absolute shrinkage and selection operator (LASSO), which is known to preserve high accuracy even when the amount of data sample is theoretically not sufficient, thus leveraging a variable selection [4]. The present study compares OLS and LASSO identification, implemented for the evaluation of measures of information transfer, as regards the detection of cardiovascular, respiratory and brain interactions during different levels of mental stress [5]. Even if LASSO regression has already been used in neuroscience, to our knowledge this is the first time in which it is introduced in a framework for the study of Information Dynamics [2] and compared with a classical approach for studying physiological interactions.

\section{MATERIALS AND METHODS}

\section{A. Data Acquisition and Pre-processing}

Data were recorded from eighteen young healthy volunteers performing three different tasks: 1) a resting condition induced by watching a relaxing video (REST);2) a stressful task obtained through mental arithmetic test (MENTAL); 3) a sustained attention task induced by following a point on a screen using the mouse (GAME).The dataset comprises seven time series, each of 300 data points, extracted from EEG, ECG, respiration and blood volume pulse signals: the heart period measuring the RR interval $(\eta)$, the respiratory amplitude $(\rho)$, the duration of blood pressure wave propagation (pulse arrival time $\operatorname{PAT}(\pi)$ ), and the EEG spectral power for the Fz electrode in the $\delta(0.5-3 \mathrm{~Hz}), \theta(4-7 \mathrm{~Hz}), \alpha(8-12$ $\mathrm{Hz})$ and $\beta(13-25 \mathrm{~Hz})$ frequency bands. More details about the experimental design and the procedures for time series extraction can be found in [5].

\section{B. Model-based analysis of Conditonal Transfer Entropy}

We consider a network with seven nodes $(M=7)$ whose activity is described by the vector stochastic process with $\mathbf{X}_{n}=\left[X_{1, n} \ldots X_{M, n}\right]^{T}$ quantifying the state of the system at the n-time step. Let us consider, $X_{j}$ as the "target" process in our network and the remaining processes $\mathbf{X}_{\mathbf{s}}$, with $\mathbf{s}=$ $\{1, \ldots, M\} \backslash j$, as "source" processes. Assuming that $\mathbf{X}$ is a Markov process of order $p$, its whole past history $\mathbf{X}_{n}^{-}$can be truncated using $p$ lags, i.e., $\mathbf{X}_{n}^{-} \simeq \mathbf{X}_{n}^{p}=\left[\mathbf{X}_{n-1}^{T} \ldots \mathbf{X}_{n-p}^{T}\right]^{T}$. In this study we consider the information transfer from the source process $X_{i}$ to the target process $X_{j}$ when the remaining source processes $\mathbf{X}_{\mathbf{k}}, \mathbf{k}=\mathbf{s} \backslash i=\{1, \ldots, M\} \backslash\{i, j\}$, are assigned, in terms of conditional transfer entropy (cTE). The cTE is a measure of the amount of information contained in the 
present state of the target that can be predicted by the past states of a specific source, above and beyond the information that is predicted already by the past states of the target and of the other sources. It is defined as follows:

$$
T_{i \rightarrow j \mid \mathbf{k}}=\ln \frac{\sigma_{j \mid j, \mathbf{k}}}{\sigma_{j \mid j, \mathbf{s}}}
$$

where $\sigma_{j \mid j, \mathbf{k}}$ and $\sigma_{j \mid j, \mathbf{s}}$ are the square root of:1) the partial variance of the target process given the past of all processes excluding the past of the source $i$ and 2) the partial variance of the target process given the past of all processes. In the present study, the variance of the target process and all the partial variances needed for the computation of the cTE were derived using the theory of SS models as described in [3]. The approach is based on describing the observed network process as VAR model of order $p$ as follows:

$$
\mathbf{X}_{n}=\sum_{k=1}^{p} \mathbf{A}_{k} \mathbf{X}_{n-k}+\mathbf{U}_{n}
$$

where $\mathbf{A}_{k}$ are the $M \times M$ matrices of coefficients and $\mathbf{U}_{n}$ is a vector of $M$ white noise Gaussian Processes. This representation is in turn expressed as a SS model, and the partial variances needed for the cTE computation are then related with the solution of the regression problem described above. A standard way to obtain the A matrix in (2) is to solve the following OLS identification problem [6]:

$$
\widehat{\mathbf{A}}=\operatorname{argmin}_{\mathbf{A}}\left\|\mathbf{X}_{n}-\mathbf{A} \mathbf{X}_{n}^{p}\right\|^{2} \text {. }
$$

After model identification, the assessment of the network topology was performed testing the significance of each estimated cTE with the method of surrogate data [5]. LASSO regression differs from OLS because it solves the following constrained problem [4]:

$$
\widehat{\mathbf{A}}=\operatorname{argmin}_{\mathbf{A}}\left\|\mathbf{X}_{n}-\mathbf{A} \mathbf{X}_{n}^{p}\right\|^{2}+\lambda\|\mathbf{A}\|_{1}
$$

where $\lambda$ is the regularization parameter that controls the amount of penalization to be applied to $\widehat{\mathbf{A}}$. As the outcome is a sparse matrix, an assessment procedure of the network in terms of statistical significance of the cTE is not needed.

\section{RESULTS}

The analysis of cTE was performed estimating the networks by means of LASSO and OLS in three different experimental conditions (REST - MENTAL - GAME). Figure 1 shows the total information transferred to each node (color-coded values of the number of subjects in which the specific link appears). The absence of color for a specific link suggests that the causal connection is absent. For each method analyzed, an evident variation in the number and location of the active connections during the different tasks is observed. The subnetwork containing the cardiovascular and respiratory time series $(\eta, \rho$ and $\pi$ ) exhibits strong connections, with a marked bidirectional coupling between $\eta$ and $\rho$ and a mainly unidirectional one from $\eta$ and $\rho$ to $\pi$. The topology of the brain subnetwork for the OLS is less stable if compared with those obtained with LASSO. The latter highlights an evident involvment of the brain subnetwork during REST with respect to GAME. Using LASSO, the analysis of the links connecting the brain and body subnetworks reveal bain $\rightarrow$ body interactions, evident particularly from brain to $\eta$ in all conditions and from brain to $\rho$ during GAME.

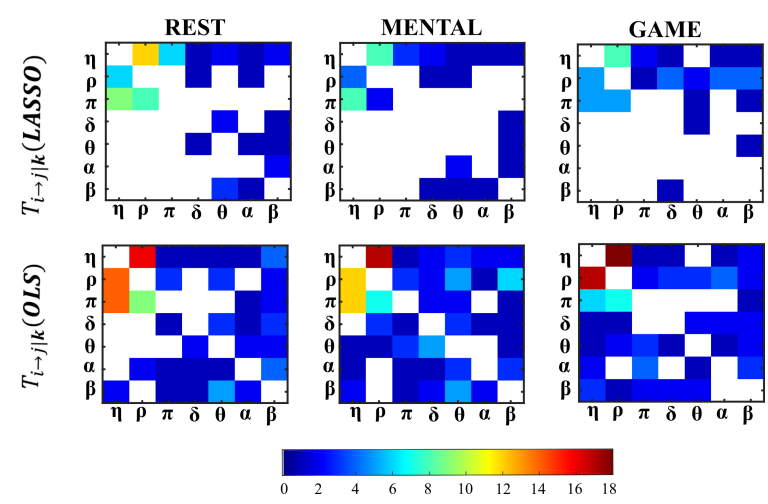

Fig. 1. Information transfer for the cardiorespiratory-brain network using the cTE. The colobar denotes the number of subjects in which the connection is statistically significant (0-white, 18 - dark red)

\section{DISCUSSION}

Our results reveal the existence of weak brain-body interactions for each mental state analyzed, confirming the results of previous works [5], [7]. Both estimation approaches highlight important cardiorespiratory interactions, while only the LASSO evidences an role of brain activity and its link with heart rate and respiration, possibly reflecting common influences from the parasympathetic neural regulation [2].

Previous works confirmed that cTE is a viable tool for the study of brain-body interactions [5] but, to our knowledge, this is the first time in which LASSO is integrated in a framework for the study of information transfer, and tested on real data for the study of brain-body interactions. Although penalized regressions have been used already to estimate causal relationships between brain signals [6], further investigations are needed to test the consistency of the methodology in the context of Network Physiology.

\section{REFERENCES}

[1] A. Bashan, "Network physiology reveals relations between network topology and physiological function," Nature communications, vol. 3, p. 702, 2012.

[2] L. Faes, "Information dynamics of brain-heart physiological networks during sleep," N. J. P., vol. 16, no. 10, p. 105005, 2014.

[3] L. Faes, "Multiscale information decomposition: Exact computation for multivariate gaussian processes," Entropy, vol. 19, no. 8, p. 408, 2017.

[4] Y. Antonacci, "Single-trial connectivity estimation through the least absolute shrinkage and selection operator," in 2019 41st Annual International Conference of the IEEE Engineering in Medicine and Biology Society (EMBC), July 2019, pp. 6422-6425.

[5] M. Zanetti, "Information dynamics of the brain, cardiovascular and respiratory network during different levels of mental stress," Entropy, vol. 21 , no. 3, p. $275,2019$.

[6] Y. Antonacci, "Estimating brain connectivity when few data points are available: Perspectives and limitations," in 2017 39th Annual International Conference of the IEEE Engineering in Medicine and Biology Society (EMBC), July 2017, pp. 4351-4354.

[7] R. Pernice, "Mutual information analysis of brain-body interactions during different levels of mental stress*," in 2019 IEEE (EMBC), July 2019, pp. 6176-6179. 